they had felt were specific to them. Furthermore, the patients considered that the film showed their symptoms to the general population in a way that should convince people that the lady in the film, with her totally relentless depression, was suffering from a real and very incapacitating illness.

The Geoffrey Knight National Unit

PAUl BRIDGes

for Affective Disorders

Brook General Hospital

London SE18 4LW

\section{Demise of the rotational training scheme}

\section{DEAR SIRS}

Implementation of Achieving a Balance is leading to the demise of the rotational training scheme. This reform is intended to prevent the bottleneck between registrar and senior registrar by shifting it to an earlier stage of doctors' careers, the step from SHO to registrar. Few would disagree with the aims of Achieving a Balance but introducing the new scheme may also have an adverse impact on training. This has certainly been the case at the Maudsley where recent changes, in keeping with Achieving a Balance, have generated controversy, ill-feeling and a loss of morale among trainees.

An important consequence of these changes has been a loss of job security for junior doctors. Among the attractions of a career in psychiatry over recent years has been the continuity, job security and commitment to training of rotational schemes. In contrast, SHO appointments in some places are now for only 12 months. Apart from increasing the stress on doctors beginning psychiatry, this may interfere with the proper balance of general and specialist posts provided by a rotation and reduce the popularity of the speciality for medical graduates.

The other concern must be what sort of criteria will be used to decide on promotion to registrar. Some rotations use passing Part I of the Membership exam as a criterion. It may prove tempting for others to use the criteria which often determined promotion through the old bottleneck to senior registrar, which placed emphasis on research publications.

This may not be an appropriate way of judging SHOs with less than a year's experience of psychiatry as it risks devaluing the clinical aspects of psychiatric training. Many trainees will wish to spend at least the first year of psychiatry increasing their knowledge beyond that expected of a medical student and finding their way around the clinical practice of psychiatry and the politics of the multidisciplinary team. One would also hope that research started after this period would be of a higher standard and of more clinical relevance.
It seems important that the College consider the implications of Achieving a Balance for the attractiveness and quality of training in psychiatry. We suggest that SHO posts should be for a minimum of two years and that full weight should be given to clinical ability in deciding upon promotion to career registrar posts.

\section{Institute of Psychiatry \\ De Crespigny Park \\ Denmark Hill \\ London SE5 8AF}

\section{ANTHONY Maden}

GLYN LEWIS

\section{Rotating junior doctors and care of the chronically mentally ill}

\section{DeAR SiRS}

In the recently published 'Statement on Approval of Training Schemes for General Professional Training for the MRCPsych' by the Royal College of Psychiatrists (Psychiatric Bulletin, February 1990, 14, $110-118$ ), the issue of the type of training is raised. This should include a minimum of one year's experience in general psychiatry, as well as at least 18 months' experience in some of the other subspecialities. The statement also suggests: "Attachments of six to 12 months' duration probably strike the best balance between the needs of training and those of the patients for continuity of care". Although widely accepted, there does not appear to have been any critical research into the relative merits and pitfalls of junior doctors rotating.

The advantages of rotations are mainly in terms of training. It allows the junior doctor to experience working for several different consultants from different backgrounds and have direct experience of some of the sub-specialities. Hopefully, these experiences are integrated so that the trainee psychiatrist has a very broad-based foundation.

However, when one views the fact that a junior doctor may be changing every six months from the viewpoint of a chronic psychiatric patient, it does raise some problems. Firstly, the trainee may be young and rather inexperienced. At first he is not going to be able properly to appreciate the course of a chronic psychiatric illness or the potential responses to treatment and there is a considerable chance that he will become very defensive in his management. If this happens, the out-patient appointment can become a rather ritualistic ceremony. Secondly, the junior doctor will inevitably lack a detailed knowledge of the individual patient. This will cause several subsidiary problems as he will not be able properly to assess what is a realistic optimal level of functioning and will be unable to balance properly the relative merits and risks of reducing or stopping medication. 
This can lead to either dangerous reductions in doses or unnecessarily prolonged periods of treatment. Finally, there is the process of developing a psychotherapeutic relationship with a very vulnerable, chronically mentally ill patient. If the patient is being seen once or twice by a different doctor every six months it is virtually impossible for such a relationship to exist. The discussion will be symptomorientated and it will be very difficult to get beyond this. In the Camberwell Study (Wing, 1982), when relatives were asked their opinions about junior doctors managing chronic patients, they pointed out that rotating doctors were poorly positioned to notice warning signals of potential relapses, tended to ask routine questions and often did not know other staff involved in the community service.

In conclusion, there appears to be somewhat of a conflict between the long-term training requirements of junior doctors and the immediate needs of the chronically mentally ill today. It is obviously important that junior psychiatrists have experience in the management of chronic patients so that they can develop skills in the prevention of relapse. However, this training needs to be organised in such a way that it does not mean that one group of patients are continually looked after by a doctor who changes every six months. Various compromises between the needs of patients and needs of training are possible. One would be ensuring that all chronic patients are jointly managed by junior doctors and permanent staff. Another would be allocating a different group of chronic patients to the care of the junior doctor every six months and taking them back at the end of that attachment. Whatever the solution, the issue does merit thinking about, researching into and emphasises the key importance of adequate supervision.

Reaside Clinic

Rubery, Rednal

Birmingham B45 9BE

\section{Reference}

Wing, J. K. (1982) (ed.) Long term community care: Experienced in a London Borough. Monograph Supplement 2: 50. Psychological Medicine.

\section{Complaints and allegations - a junior doctor perspective}

DEAR SIRS

According to a major insurer, the frequency of malpractice claims in the USA has risen from 10.5 claims per 100 physicians in 1980 to 17.8 claims per 100 in 1986 (St Paul Fire and Marine Insurance Company, 1986). In the UK in addition to an increase in litigation there has also been an increase in the number of complaints that has reached health authorities within the NHS.

The 'Malpractice Stress Disorder' has been described in the USA (Reading, 1986); it refers to the emotional and behavioural responses of those being sued. Doctors experience allegations as a direct assault on their sense of self. As a result of litigation physicians have experienced some disruption in relation to their role and to their interaction with others (Charles et al, 1988). Charles et al (1984) found one group showed symptoms of clinical depression including suicidal thoughts while another had overwhelming anger with difficulties in making decisions and a general feeling of dissatisfaction. In one case a doctor committed suicide as a result of litigation (Foulkes, 1987).

It seems that the content and circumstances of allegations have little influence on the nature of the reaction and one study found no significant differences in symptoms between physicians who won and those who lost their trial (Charles et al, 1985).

To my knowledge no similar research has been carried out in the UK. My personal observations of colleagues troubled by complaint procedures are that their self-esteem is reduced and they show irritability, anxiety and low mood. The news reaches other departments, hospitals and even other districts; rumour spreads and the colleague is pitied. This lowers self-esteem further. While the matter remains unresolved the doctor has to perform as if nothing has happened. When work performance suffers this in turn lowers self-confidence.

As the junior doctor wants to sit a postgraduate exam the investigation procedures present a burden that inevitably have a negative effect on performance. A failure in Membership exams in such situations has a much more devastating effect than it would have under normal circumstances.

I feel that the investigating body has a responsibility and obligation towards the victim of allegations for as long as investigations proceed which should result in concrete help. I would propose that, as soon as an investigation is initiated, a counsellor is identified who will accompany the doctor while these procedures are ongoing. I would hope that this support will enable the colleague to talk freely and ventilate his or her feelings and have a positive effect on self-esteem and self-confidence.

\section{Regional Brain Injury \\ Rehabilitation Unit \\ St Albans \\ Hertfordshire AL2 1BR}

\section{References}

Charles, S. C., Wilbert, J. R. \& Kennedy, E. C. (1984) Physicians' self-reports of reactions to malpractice litigation. The American Journal of Psychiatry, 141, 563-565. 